\title{
Contribuição ao diagnóstico morfológico da adiaspiromicose pulmonar
}

\author{
Contribution to morphologic diagnosis of pulmonary adiaspiromycosis
}

\author{
Vitorino Modesto dos Santos ${ }^{12}$, Marlene Antonia dos Reis', Sheila Jorge Adad', \\ João Carlos Saldanha' e Vicente de Paula Antunes Teixeira' ${ }^{1}$
}

\begin{abstract}
Resumo Adiaspiromicose é usualmente diagnosticada em tecido pulmonar corado por hematoxilina-eosina, ácido periódico Schiff e prata-metenamina. Os autores descrevem a morfologia do fungo corado pelo mucicarmim, picro-sírius e vermelho Congo, inclusive à luz polarizada. Tratando-se de diagnósticos duvidosos, essas técnicas poderiam facilitar na diferenciação entre Emmonsia parva var crescens e outros agentes.
\end{abstract}

Palavras-chaves: Micoses pulmonares. Adiaspiromicose. Emmonsia parva var crescens. Chrysosporium parvum var crescens.

\begin{abstract}
The diagnosis of adiaspiromycosis is usually based on lung sections stained by hematoxylin-eosin, periodic acid Schiff and methenamine silver. Authors describe the fungus aspect examined by mucicarmin, picro-sirius and Congo red methods, including polarized light microscopy. In doubtful cases, these methods could contribute to histopathological diagnosis of Emmonsia parva var crescens.
\end{abstract}

Key-words: Pulmonary mycosis. Adiaspiromycosis. Emmonsia parva var crescens. Chrysosporium parvum var crescens.

Adiaspiromicose $(\mathrm{a}=$ não + diaspeiros $=$ disseminar, distribuir, dispersar, espalhar, difundir + mykez $=$ fungo $)^{12}$ significa ausência de multiplicação e de disseminação nos hospedeiros, embora o fungo tenha sido descrito nos seios paranasais, encéfalo e rim de paciente com leucemia ${ }^{15}$, no rádio de paciente com $\operatorname{AIDS}^{6}$ e em linfonodos traqueobrônquicos e mediastinais de cangambá ${ }^{2}$. Predomina em climas temperados, sendo freqüente em pulmões de roedores que cavam e vivem em tocas ${ }^{11}$. Animais de rapina eliminam o fungo nas fezes, contaminando o solo, onde se desenvolve e pode ser inalado. Nos pulmões de hospedeiros, cada adiaconídio representa um propágulo 71320 .
A doença é causada por Emmonsia. parva var. crescens ${ }^{14} 172024$ ou Chrysosporium parvum var. crescens $^{1326}$. Embora persistam controvérsias ${ }^{4}{ }^{1424}$, segundo Ajello ${ }^{13}$ o fungo pertence à divisão: Eumycota; subdivisão: Deuteromycotina; classe: Hyphomycetes; gênero: Emmonsia; espécie: parva; variedade crescens. Estudos de biologia molecular relacionam Emmonsia ao gênero Ajellomyces, família Onygenaceae, a que pertencem fungos patogênicos como Blastomyces dermatitidis e Histoplasma capsulatum ${ }^{4} 142024$.

Emmonsia é um fungo dimórfico cujos aleuroconídios, medindo $2-4 \mu \mathrm{m}^{1926}$, transformamse em esférulas multinucleadas de paredes espessas (adiaconídios) que crescem até

\footnotetext{
1. Curso de Pós-graduação em Patologia; 2. Departamento de Clínica Médica da Faculdade de Medicina do Triângulo Mineiro (FMTM), Uberaba, MG.

Apoio financeiro: Fundação de Ensino e Pesquisa de Uberaba (FUNEPU), Uberaba, MG.

Endereço para correspondência: Prof. Vitorino Modesto dos Santos. Al. República 64, Recanto das Torres, 38057-020, Uberaba, MG. Fax: 55343185335

Recebido para publicação em 31/3/99.
} 
$700 \mu \mathrm{m}^{378131922}$, provocando resposta inflamatória no sítio de infecção². Há grande variabilidade no número, tamanho, intensidade de coloração e quantidade de citoplasma entre os fungos presentes nos granulomas ${ }^{7}$. Em cortes tangenciais podem ter aspecto sólido; mas, geralmente apresentam-se como estruturas anelares vazias, ou com pequenos glóbulos hialinos refrativos e finos grânulos basofílicos ao longo da parede interna, sem qualquer evidência de multiplicação ${ }^{2726}$.

Cerca de metade dos casos relatados de adiaspiromicose humana ocorreram no Brasil ${ }^{9}$. Tem sido descrita sua associação com uso de imunossupressores, abscessos pulmonares, aspergilose, bronquiectasias, candidíase, coccidioidomicose, criptococose, doença pulmonar obstrutiva crônica, linfomas, leucemia, tuberculose e AIDS 56791315 . Além dos pulmões, E. parva foi descrita em prótese valvular cardíaca, lesões cutâneas e apendicite complicada ${ }^{18}$.

A gravidade das lesões pulmonares e 0 quadro clínico dependem do número de conídios inalados ${ }^{1925}$, de enfermidades associadas, da antigenicidade do fungo ${ }^{2} \mathrm{e}$ da resposta imune do hospedeiro. A concomitância de lesões pulmonares cavitárias coalescentes e supurativas (de semanas a poucos meses), com granulomas epitelióides e fibrose hialinizada (de vários meses a anos) sugere que a inoculação do fungo pode repetirse em diferentes ocasiões ${ }^{18}{ }^{22}$.

Para definição diagnóstica, utiliza-se a biópsia transbrônquica ${ }^{16}$ (fragmentos $<2 \mathrm{~mm}$ ) e o lavado broncoalveolar, métodos que nem sempre permitem definir a etiologia de pequenos nódulos ou infiltrados intersticiais. Nessa circunstância, preconiza-se ${ }^{22}$ a biópsia por videotoracoscopia, por ser menos invasiva, fornecer maiores fragmentos de tecido, com morbidade e mortalidade mínimas, tendo eficácia comparável à da toracotomia ${ }^{10}$. A adiaspiromicose localizada usualmente tem remissão espontânea; porém, drogas antifúngicas são utilizadas na forma pulmonar disseminada 16171920222325 que pode evoluir com insuficiência respiratória grave ${ }^{4}$, inclusive causando o óbito ${ }^{18} 21$.

O diagnóstico é estabelecido observando-se adiaconídios em amostras de tecido pulmonar coradas por hematoxilina-eosina (HE), pratametenamina (Grocott ou Gomori) e ácido periódico Schiff (PAS) ${ }^{226}$. Adiaconídios menores, mais jovens, coram-se mais intensamente. $O$ aspecto da parede celular varia dependendo da coloração utilizada $^{26}$. No HE (Figuras 1 e 2), apresentam membrana celular espessa (10-90 $\mu \mathrm{m})$ e bilaminar, constituída por uma área interna pouco corada, homogênea, vítrea, levemente basofílica, pouco refrativa, não birrefringente, circundada por uma zona externa larga, intensamente eosinofílica onde se notam fenestrações. A membrana mostra-se trilaminar quando corada pelo PAS (Figura 3) e é intensamente impregnada pela prata-metenamina (Figuras 4 e 5) que acentua a presença de fenestrações ${ }^{926}$.

Pelo mucicarmim (Figura 6A), as camadas interna e média da parede mostram-se discretamente positivas, indicando a presença de muco ácido e neutro, sem birrefringência à luz polarizada (Figura 6B). Semelhante ao que foi descrito com relação a Paracoccidioides brasiliensis, empregando-se a técnica do picro-sirius ${ }^{1}$, a parede do adiaconídio mostra birrefringência de cor alaranjada à luz polarizada (Figuras 7A e 7B). Utilizando-se o vermelho Congo notam-se mais de três camadas na parede, com intensa birrefringência amarelada à luz polarizada (Figuras 8A e 8B). A microscopia com contraste de fase, revela com nitidez a estrutura trilaminar da parede, mesmo em cortes corados pela hematoxilina-eosina (Figuras 9A e 9B).

Na investigação diagnóstica de rotina não têm sido úteis as culturas, os testes cutâneos, nem a inoculação em animais de laboratório ${ }^{20}$. Embora menos sensível e específico que os exames micológicos, o estudo histopatológico permite comprovar a invasão dos tecidos pelo fungo. $\mathrm{Na}$ avaliação histopatológica, entretanto, constatase grande variabilidade nas características dos adiaconídios descritos nos granulomas, incluindo número, forma, tamanho $(2-700 \mu \mathrm{m})$, estrutura da parede, quantidade de citoplasma, intensidade de coloração e aspecto à luz polarizada. Esse fato pode propiciar equívocos diagnósticos entre Emmonsia parva e outros agentes que em maior número e com maior freqüência acometem os pulmões, em especial quando estiverem associados a adiaconídios de menor tamanho.

Fatores que dificultam o diagnóstico de adiaspiromicose incluem o conhecimento muito limitado sobre a doença, a concomitância de infecção por outros fungos (Aspergyllus, Cândida, Coccidioides, Cryptococcus) $)^{79}$, infecções assintomáticas e manifestações clínicas inespecíficas, aspecto radiológico compatível com outras pneumopatias, falta de teste sorológico específico e possibilidade de reações cruzadas com outros fungos (Blastomyces, Coccidioides, 


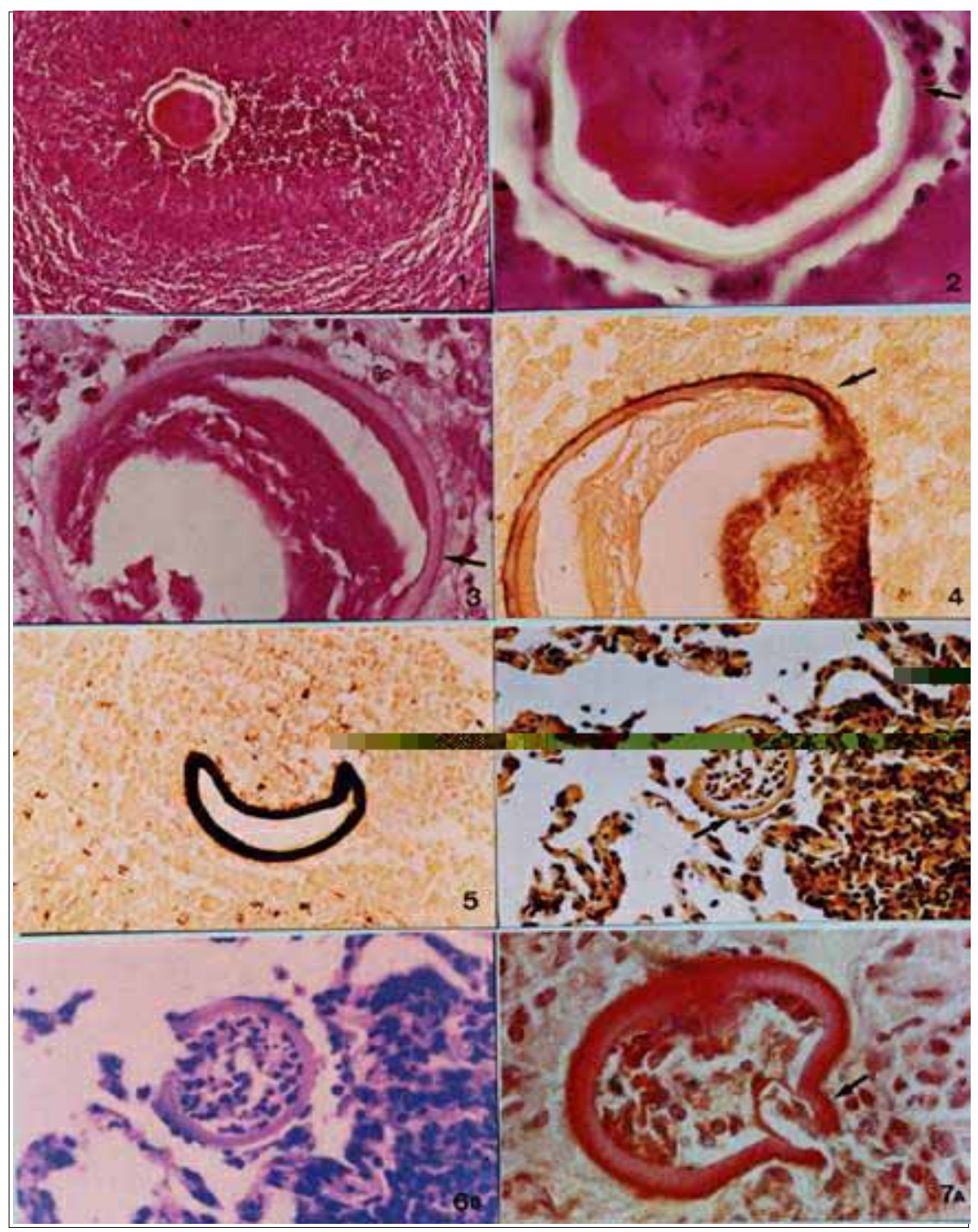

Figuras. Cortes histológicos de pulmão com granulomas contendo adiaconídios de Emmonsia parva corados por vários métodos histoquímicos.

Figura 1 - Adiaconídio envolto por neutrófilos, macrófagos e linfoplasmócitos. HE x 250.

Figura 2 - Detalhe do adiaconídio, mostrando (seta) aspecto bilaminar da parede. HE x 1250. Figura 3 - Aspecto trilaminar (seta) da parede. PAS x 1250.

Figura 4 - Parede trilaminar (seta) em adiaconídio bem preservado. Prata-metenamina x 1250. Figura 5 - Adiaconídio colapsado mostrando-se intensamente corado. Prata-metenamina x 500 Figura 6 - A) Camada interna da parede (seta) discretamente corada. Mucicarmim x 500. B) À luz polarizada, a parede não apresenta birrefringência. Mucicarmim x 500.

Figura 7A - Adiaconídio apresentando (seta) parede trilaminar. Picro-sirius x 1250. 
Histoplasma, Paracoccidioides) ${ }^{11}$, cultura do fungo não disponível, amostras de tecido inadequadas para exame e possibilidade de cura espontânea na maioria dos casos. Com o processo inflamatório, os adiaconídios podem sofrer acentuadas modificações de forma e tamanho, simulando a presença de outros fungos (Blastomyces, Coccidioides, Curvularia, Rhinosporidium), helmintos (Strongyloides, Dirofilaria), grãos de pólen e partículas minerais ou vegetais inalados 392226 (Figura 10).

Após o primeiro relato de adiaspiromicose pulmonar em paciente do Hospital Escola da FMTM $^{22}$ mais dois casos foram confirmados em nosso meio, sugerindo a possibilidade de que a real freqüência dessa infecção esteja sendo subestimada.

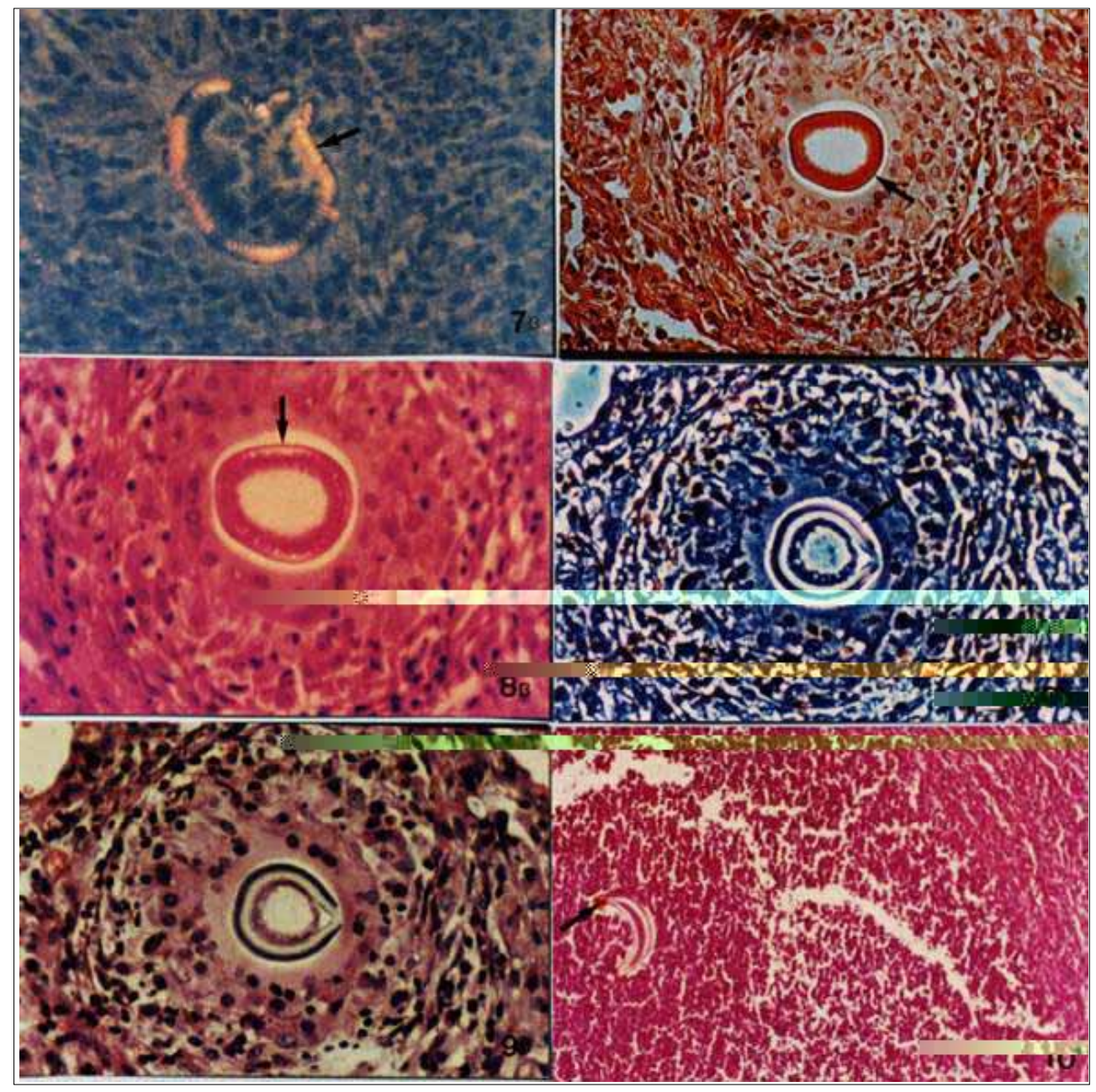

Figura 7B - À luz polarizada, parede birrefringente (seta) de cor alaranjada. Picro-sirius x 1250 .

Figura 8 - A) Parede de adiaconídio (seta) com mais de três camadas. Vermelho Congo x 500. B) Birrefringência de cor amarelada (seta) à luz polarizada. Vermelho Congo x 500.

Figura 9 - A) Maior nitidez das estruturas e parede trilaminar (seta) ao contraste de fase. HE x 625. B) Aspecto do fungo da figura anterior, à microscopia de luz comum. HE x 625 .

Figura 10 - Adiaconídio (seta) com aspecto de larva de Strongyloides stercoralis. HE x 250. 


\section{AGRADECIMENTOS}

Os autores agradecem às técnicas Valéria Ramos e Jucélia Ribeiro Torres pela confecção dos preparados histológicos e à FUNEPU pelo apoio financeiro.

\section{REFERÊNCIAS BIBLIOGRÁFICAS}

1. Almeida HO, Teixeira VPA, Gobbi H, Morais MGR, Reis MA, Araújo MBM. Aspectos do Cryptococcus neoformans e do Paracoccidioides brasiliensis corados pelo picro-sírius. Revista Latinoamericana de Microbiologia 32:6-10, 1990.

2. Albassam MA, Bhatnagar R, Lillie LE, Roy L. Adiaspiromycosis in striped skunks in Alberta, Canada. Journal of Wildlife Diseases 22:13-18, 1986.

3. Barbas Filho JV, Amato MB, Deheinzelin D, Daldiva $\mathrm{PH}$, Carvalho CR. Respiratory failure caused by adiaspiromycosis. Chest 97:1171-1175, 1990.

4. Bowman BH, White TJ, Taylor JW. Human pathogenetic fungi and their close nonpathogenic relatives. Molecular Phylogenetics and Evolution 6:89-96, 1996.

5. Doby-Dubois M, Chevrel ML, Doby JM, Louvet M. Premier cas humain d'adiaspiromycose, par Emmonsia crescens, Emmons et Jellison, 1960. Bulletin de la Sociétè de Pathologie Exotique 57:240-244, 1964.

6. Echavarria E, Cano EL, Restrepo A. Disseminated adiaspiromycosis in a patient with AIDS. Journal of Medical and Veterinary Mycology 31: 91-97, 1993.

7. Emmons CW, Ashburn LL. The isolation of Haplosporangium parvum n. sp.and Coccidioides immitis from wild rodents. Their relationship to coccidioidomycosis. Public Health Reports 57:1715-1727, 1942.

8. Emmons CW, Jellison WL. Emmonsia crescens $\mathrm{sp} n$ and adiaspiromycosis (Haplomycosis) in mammals. Annals New York Academy of Sciences 89:91-101, 1960.

9. England DM, Hochholzer L. Adiaspiromycosis: an unusual fungal infection of the lung. Report of 11 cases. The American Journal of Surgical Pathology 17:876-886, 1993.

10. Ferson PF, Landreneau RJ, Dowling RD, Hazebrigg SR, Ritter P, Nunchuck S, Perrino MK, Bowers CM, Mack MJ, Magee MJ. Comparison of open versus thoracoscopic lung biopsy for diffuse infiltrative pulmonary disease. Journal of Thoracic Cardiovascular Surgery 106:194-196, 1993.

11. Hubalek Z, Nesvadbova J, Halouzka J. Emmonsiosis of rodents in an agroecosystem. Journal of Medical Mycology 36:387-390, 1998.

12. Isidro Pereira SJ. Dicionário Grego-Português e Português-Grego. $7^{a}$ edição. Editorial A.L., Braga, 1990.

13. Lacaz CS, Porto E, Martins JEC. Micologia Médica. $8^{\text {a }}$ edição. Sarvier, São Paulo, 1991.

14. Leclerc MC, Philippe H, Guého E. Phylogeny of dermatophytes and dimorphic fungi based on large subunit ribosomal RNA sequence comparisons. Journal of Medical and Veterinary Mycology 32:331-341, 1994.

15. Levy FE, Larson JT, George E, Maisel RH. Invasive chrysosporium infection of the nose and paranasal sinuses in an immunocompromised host. OtolaryngologyHead and Neck Surgery 104:384-388, 1991.

16. Lima TSM, Moraes MAP, Magalhães HQ, Athayde NSG. Novo caso de adiaspiromicose humana diagnosticado por biópsia transbrônquica. Jornal de Pneumologia 24:339-341, 1998.

17. Martins RLM, Santos CGF, França FRFC, Moraes MAP. Adiaspiromicose humana. Relato de um caso tratado com cetoconazol. Revista da Sociedade Brasileira de Medicina Tropical 30:507-509, 1997.

18. Moraes MAP, Almeida MC, Raick AN. Caso fatal de adiaspiromicose pulmonar humana. Revista do Instituto de Medicina Tropical de São Paulo 31:188-194, 1989.

19. Moraes MAP, Silva AE, Raick AN. Adiaspiromicose pulmonar humana. Novo caso da forma disseminada. Revista da Sociedade Brasileira de Medicina Tropical 23:171-174, 1990.

20. Nuorva K, Pitkänen R, Issakainen J, Huttunen NP, Juhola M. Pulmonary adiaspiromycosis in a two year old girl. Journal of Clinical Pathology 50:82-85, 1997.

21. Peres LC, Figueiredo F, Peinado M, Soares FA. Fulminant disseminated pulmonay adiaspiromycosis in humans. The American Journal of Tropical Medicine and Hygiene 46:146-150, 1992.

22. Santos VM, Santana JH, Adad SJ, Lopes GP, Fatureto MC. Adiaspiromicose pulmonar disseminada. Relato de caso. Revista da Sociedade Brasileira de Medicina Tropical 30:397-400, 1997.

23. Severo LC, Geyer GR, Camargo JJ, Porto NS. Adiaspiromycosis treated successfully with ketoconazole. Journal of Medical and Veterinary Mycology 27:265-268, 1989.

24. Sigler L. Ajellomyces crescens sp. nov., taxonomy of Emmonsia spp, and relatedness with Blastomyces dermatitidis (teleomorph Ajellomyces dermatitidis). Journal of Medical and Veterinary Mycology 34:303-314, 1996.

25. Teixeira AA, Moraes RC, Magalhães $H Q$, Bumbieris ES. Adiaspiromicose pulmonar granulomatosa disseminada. Radiologia Brasileira 24:121-126, 1991.

26. Watts JC, Chandler FW. Adiaspiromycosis. In: Connor DH (ed) Pathology of infectious diseases. Appleton \& Lange, Stamford, p. 929-932, 1997. 